\section{Available at \\ www.ElsevierMathematics.com \\ POWERED B S IENCE d DiREct.}

Ann. I. H. Poincaré - PR 40 (2004) 43-52

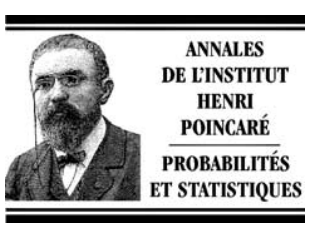

www.elsevier.com/locate/anihpb

\title{
A note on the two-sided regulated random walk
}

\author{
A. Manita ${ }^{a}$, F. Simonot ${ }^{b}$ \\ a Department of Probability, Faculty of Mechanics and mathematics, Moscow State University, Moscow 119899, Russia \\ b IECN, Université Henri Poincaré, Nancy 1 et Esstin, rue Jean Lamour, 54500 Vandoeuvre, France \\ Received 25 July 2002; accepted 27 June 2003
}

\begin{abstract}
In this paper we address the two-sided regulated random walk defined by the relation $X_{N}(t+1)=\min \left(N, \max \left(0, X_{N}(t)+\right.\right.$ $A(t+1)))$ where $(A(t) ; t \geqslant 1)$ is a sequence of i.i.d r.v's with integer values such that $A(t) \geqslant-1, E\{A\}=0$ and $E\left\{r^{A}\right\}<+\infty$ for an $r>1$. Denoting by $\pi_{N}$ its stationary distribution, $F_{N}(x)=\pi_{N}([0, N x])$ and $G(x)$ the d.f of a uniform r.v on [0,1]. It is shown that $0<\underline{\lim } N\left\|F_{N}-G\right\|_{p} \leqslant \varlimsup \lim N\left\|F_{N}-G\right\|_{p}<+\infty$ for $1 \leqslant p \leqslant+\infty$, that is: $1 / N$ is the exact convergence rate of $F_{N}$ to $G$. This result improves (in the particular case considered) earlier results claiming that $\lim _{N}\left\|F_{N}-G\right\|_{\infty}=0$. (c) 2003 Elsevier SAS. All rights reserved.
\end{abstract}

\section{Résumé}

Cet article considère la marche aléatoire doublement régulée, définie par la relation de récurrence $X_{N}(t+1)=$ $\min \left(N, \max \left(0, X_{N}(t)+A(t+1)\right)\right)$ où $(A(t) ; t \geqslant 1)$ est une suite de v.a entières i.i.d vérifiant $A(t) \geqslant-1, E\{A\}=0$ et $E\left\{r^{A}\right\}<+\infty$ pour un $r>1$. Notant $\pi_{N}$ sa distribution stationnaire, $F_{N}(x)=\pi_{N}([0, N x])$ et $G(x)$ la f.r d'une v.a uniforme sur $[0,1]$, nous montrons que $0<\underline{\lim } N\left\|F_{N}-G\right\|_{p} \leqslant \varlimsup \lim N\left\|F_{N}-G\right\|_{p}<+\infty$ pour tout $p$ tel que $1 \leqslant p \leqslant+\infty$. C'est à dire que $1 / N$ est le taux exact de convergence de $F_{N}$ vers $G$. Ce résultat améliore (dans le cas particulier considéré) un résultat antérieur affirmant que $\lim _{N}\left\|F_{N}-G\right\|_{\infty}=0$.

(c) 2003 Elsevier SAS. All rights reserved.

$M S C$ : primary $60 \mathrm{~J} 10,60 \mathrm{~K} 25$; secondary $60 \mathrm{~F} 25,60 \mathrm{~F} 99$

Keywords: Markov chain; Regulated random walk; Stochastic ordering; Convergence rate

\section{Introduction}

This paper deals with the asymptotic behaviour of the stationary distribution $\pi_{N}$ of the two-sided regulated random walk as the upper boundary $N$ tends to infinity. Specifically, we consider a sequence $(A(t) ; t \geqslant 1)$ of i.i.d r.v's with values in $Z$ and for any integer $N$, the attached Markov chain $\left(X_{N}(t) ; t \geqslant 0\right)$ defined recursively by the equation

$$
X_{N}(t+1)=\min \left(N, \max \left(0, X_{N}(t)+A(t+1)\right)\right) \quad \text { for } t \geqslant 0 ; X_{N}(0) \in\{0,1, \ldots, N\} .
$$

E-mail addresses: manita@mech.math.msu.su (A. Manita), francois.simonot@esstin.uhp-nancy.fr (F. Simonot). 
The TPM (Transition Probability Matrix) $P_{N}=\left(p_{N}(i, j)\right)_{0 \leqslant i, j \leqslant N}$ of which being given by

$$
P_{N}=\left[\begin{array}{ccccccc}
\tilde{a}_{0} & a_{1} & a_{2} & \cdots & \cdots & a_{N-1} & \bar{a}_{N} \\
\tilde{a}_{-1} & a_{0} & a_{1} & \cdots & \cdots & a_{N-2} & \bar{a}_{N-1} \\
\tilde{a}_{-2} & a_{-1} & a_{0} & \cdots & \cdots & a_{N-3} & \bar{a}_{N-2} \\
\vdots & \vdots & \ddots & \ddots & \ddots & \vdots & \vdots \\
\vdots & \vdots & \ddots & \ddots & \ddots & \vdots & \vdots \\
\tilde{a}_{-(N-1)} & a_{-(N-2)} & a_{-(N-3)} & \ddots & \ddots & a_{0} & \bar{a}_{1} \\
\tilde{a}_{-N} & a_{-(N-1)} & a_{-(N-2)} & \ddots & \ddots & a_{-1} & \bar{a}_{0}
\end{array}\right],
$$

where $\tilde{a}_{k}=\sum_{j \leqslant k} a_{j}$ and $\bar{a}_{k}=\sum_{j \geqslant k} a_{j}$.

Following the terminology of [4], $\left(X_{N}(t) ; t \geqslant 0\right)$ is called the two-sided regulated random walk, the expression reflected random walk being reserved to the case when $p_{N}(0,1)=1$ (reflexion at level 0 ) or $p_{N}(N, N-1)=1$ (reflexion at level $N$ ).

The main motivation of the present paper is due to the fact that equation (1.1) frequently appears in stochastic modelling and applied probability topics such as queueing, storage and various communication systems with finite capacity (see for instance $[1,2,5,10,11,16,17])$. Together with $\left(X_{N}(t) ; t \geqslant 0\right)$, we can consider the Markov chain $(X(t) ; t \geqslant 0)=\left(X_{\infty}(t) ; t \geqslant 0\right)$ corresponding to $N=+\infty$, obviously defined by

$$
X(t+1)=\max (0, X(t)+A(t+1)) \text { for } t \geqslant 0 ; X(0) \geqslant 0,
$$

which is called the one-sided regulated random walk regulated at level 0 or sometimes "Lindley process".

\subsection{Asymptotic behaviour of the stationary distribution $\pi_{N}$ of $\left(X_{N}(t) ; t \geqslant 0\right)$}

To place this problem in its context let's recall some results. Assuming $(X(t) ; t \geqslant 0)$ and $\left(X_{N}(t) ; t \geqslant 0\right)$ irreducible, let's consider the following cases:

(1) If $E\{A\}=\mu<0$, it is known that both $\left(X_{N}(t) ; t \geqslant 0\right)$ and $\left(X_{\infty}(t) ; t \geqslant 0\right)$ admit limiting distribution $\pi_{N}$ and $\pi$ respectively and that:

$$
\lim _{N} \sum_{k \geqslant 0}\left|\pi_{N}(k)-\pi(k)\right|=0
$$

more or less rapidly depending on the additional assumptions made on the increment $A$, this case has been investigated in many papers such as $[3,6-8,12-14,18,19]$.

(2) If $E\{A\}=\mu>0$, letting $Y_{N}(t)=N-X_{N}(t)$, it is readily seen that $\left(Y_{N}(t) ; t \geqslant 0\right)$ obeys the relation

$$
Y_{N}(t+1)=\min \left(N, \max \left(0, Y_{N}(t)-A(t+1)\right)\right) \quad \text { for } t \geqslant 0 ; Y_{N}(0) \in\{0,1, \ldots, N\}
$$

therefore the result of (1) applies to $\left(Y_{N}(t) ; t \geqslant 0\right)$ and the properties of $X_{N}(t)$ can be derived from $X_{N}(t)=N-Y_{N}(t)$.

In the present paper we address the asymptotic behaviour of the stationary distribution $\pi_{N}$ of $\left(X_{N}(t) ; t \geqslant 0\right)$, when $E\{A\}=0$. By now, it is worth noticing that the limiting distribution $\pi_{N}$ always exists but $(X(t) ; t \geqslant 0)$ no longer admits a stationary distribution, therefore we can't hope a result similar to (1.4). But as will be seen in the sequel the limiting distribution of the renormalized Markov chain $\left(N^{-1} X_{N}(t) ; t \geqslant 0\right)$ weakly converges to a uniform distribution as $N$ tends to infinity. 


\subsection{Previous work}

As far as we know, a previous result could be Theorem 1 below, which is something like a "folk theorem", since on one hand it seems to be known, at least intuitively, on the ground of similar result about the two-sided regulated brownian motion (see $[2,4]$ ) and on the other hand, we were unable to find any clear and explicit reference in the litterature. A proof can be found in [15], Lemma 10.

From now on, we denote by $G$ be the d.f of a uniform r.v on $[0,1]$.

Theorem 1. If $E\{A\}=0, E\left\{A^{2}\right\}<+\infty$ and $\left(X_{N}(t) ; t \geqslant 0\right)$ is irreducible then

$$
\limsup _{N}\left|\pi_{N}([0, N x])-G(x)\right|=0
$$

\subsection{Main results}

We intend to improve the Theorem 1 when $(A(t) ; t \geqslant 1)$ obeys additional assumptions frequently met in applications (see the examples). Defining $a_{m}=P(A(t)=m)$, from now on it will be assumed that

(AS1) $P(A<-1)=0, a_{-1}>0, a_{0}+a_{-1}<1$;

(AS2) $E\{A\}=0$

(AS3) $\exists r>1, E\left(r^{A}\right)<+\infty$.

Under the above-mentioned assumptions, it turns out that

\section{Property 1.}

(i) $\varlimsup \lim N \sum_{0}^{N}\left|\pi_{N}(n)-\frac{1}{N+1}\right|<+\infty$.

(ii) If $\underline{\lim } N \sum_{0}^{N}\left|\pi_{N}(n)-\frac{1}{N+1}\right|=0$, then for any $N, \pi_{N}$ is uniform on $\{0,1,2, \ldots, N\}$.

As a consequence, letting $F_{N}(x)=\pi_{N}([0, N x])$ and denoting as usual $\|f\|_{\infty}=\sup _{x}|f(x)|$ and $\|f\|_{p}^{p}=$ $\int|f(x)|^{p} \mathrm{~d} x$ for $p \geqslant 1$, we arrive at

\section{Theorem 2.}

$$
\frac{\sigma^{2}}{2 a_{-1}} \leqslant \underline{\lim } N\left\|F_{N}-G\right\|_{\infty} \leqslant \varlimsup \lim N\left\|F_{N}-G\right\|_{\infty}<+\infty .
$$

\section{Theorem 3.}

$$
\frac{1}{2}(p+1)^{-p^{-1}} \leqslant \underline{\lim } N\left\|F_{N}-G\right\|_{p} \leqslant \varlimsup
$$

In any case, $\frac{1}{N}$ is the exact convergence rate. 


\section{Proofs}

Under (AS1), (AS2), (AS3), the TPM of the two-sided regulated random walk $\left(X_{N}(t) ; t \geqslant 0\right)$ defined by (1.1) can be written down as

$$
P_{N}=\left[\begin{array}{ccccccc}
\tilde{a}_{0} & a_{1} & a_{2} & \cdots & \cdots & a_{N-1} & \bar{a}_{N} \\
a_{-1} & a_{0} & a_{1} & \cdots & \cdots & a_{N-2} & \bar{a}_{N-1} \\
0 & a_{-1} & a_{0} & \cdots & \cdots & a_{N-3} & \bar{a}_{N-2} \\
0 & 0 & \ddots & \ddots & \ddots & \vdots & \vdots \\
\vdots & \vdots & \ddots & \ddots & \ddots & \vdots & \vdots \\
0 & 0 & 0 & \ddots & \ddots & a_{0} & \bar{a}_{1} \\
0 & 0 & 0 & \ddots & \ddots & a_{-1} & \bar{a}_{0}
\end{array}\right],
$$

where $\tilde{a}_{0}=a_{0}+a_{-1}$ and $\bar{a}_{k}=\sum_{j \geqslant k} a_{j}$.

First, we notice that since $a_{-1}>0, a_{0}+a_{-1}<1, P_{N}$ is irreducible, therefore the limiting distribution $\pi_{N}$ exists and is also the unique stationary distribution given by $\pi_{N}=\pi_{N} P_{N}$ with $\sum_{0 \leqslant n \leqslant N} \pi_{N}(n)=1$.

\subsection{An explicit calculation of $\pi_{N}$}

Based on a result of Korolyuk [9] an explicit analytic expression for $\pi_{N}(n)$ is derived. Namely, the stationary distribution of the Markov chain $\left(X_{N}(t) ; t \geqslant 0\right)$ is given by the following equations:

$$
\begin{aligned}
& \pi_{N}(0)=a_{-1} \pi_{N}(1)+\left(a_{0}+a_{-1}\right) \pi_{N}(0), \\
& \pi_{N}(1)=a_{-1} \pi_{N}(2)+a_{0} \pi_{N}(1)+a_{1} \pi_{N}(0), \\
& \ldots \\
& \pi_{N}(n)=a_{-1} \pi_{N}(n+1)+a_{0} \pi_{N}(n)+\cdots+a_{n-1} \pi_{N}(1)+a_{n} \pi_{N}(0), \\
& \ldots \\
& \pi_{N}(N-1)=a_{-1} \pi_{N}(N)+a_{0} \pi_{N}(N-1)+\cdots+a_{N-2} \pi_{N}(1)+a_{N-1} \pi_{N}(0), \\
& \pi_{N}(N)=\bar{a}_{0} \pi_{N}(N)+\bar{a}_{1} \pi_{N}(N-1)+\cdots+\bar{a}_{N-1} \pi_{N}(1)+\bar{a}_{N} \pi_{N}(0),
\end{aligned}
$$

with the condition $\sum_{0 \leqslant n \leqslant N} \pi_{N}(n)=1$.

Let's introduce some notations:

$$
G(z)=E\left(z^{A}\right)=\sum_{k \geqslant-1} a_{k} z^{k}, \quad D(z)=z G(z)-z, \quad h(z)=\frac{z}{D(z)}=\sum_{k \geqslant 1} B_{k} z^{k} .
$$

The assumptions $E(A)=0$ and $E\left(A^{2}\right)=\sigma^{2}$ entail $D(1)=D^{\prime}(1)=0$ and $D^{\prime \prime}(1)=\sigma^{2}$. Due to the assumption (AS3), it is seen that there exists a number $\rho>1$ for which $D(z)$ is analytic in $U=\{z:|z|<\rho\}$. Now $h(z)$ is analytic everywhere in $U=\{z:|z|<\rho\}$ except for the zeros of $D(z)$, but it can be easily proved that if $D(z)=0$ then $|z|>1$ or $z=1$. It turns out that we can find an $R>1$ for which $h(z)$ is analytic in $V=\{z:|z|<R\}-\{1\}$ and thus admits a Taylor's expansion $h(z)=\sum_{k \geqslant 1} B_{k} z^{k}$ in a neighbourhood of zero. We are now ready to search for $\pi_{N}$. This is done with:

Lemma 1 [9]. Let $h(z)=\sum_{k \geqslant 1} B_{k} z^{k}$ then, for $1 \leqslant n \leqslant N$,

$$
\pi_{N}(n)=\left(1+\sum_{k=1}^{n-1} B_{k} \bar{a}_{n-k+1}+B_{n}\left(\bar{a}_{1}-a_{-1}\right)\right) \pi_{N}(0)
$$

and $\pi_{N}(0)$ is defined by the condition $\sum_{0 \leqslant n \leqslant N} \pi_{N}(n)=1$. 
Proof. Is achieved by direct substitution.

We proceed further by giving a more detailed expression of $\pi_{N}$ showing that $\pi_{N}(n) / \pi_{N}(0)$ is "almost" constant. The Laurent's expansion of $h$ in a neighbourhood of 1 can be written down as

$$
h(z)=\frac{\alpha_{-2}}{(1-z)^{2}}+\frac{\alpha_{-1}}{1-z}+\sum_{k \geqslant 0} \alpha_{k}(z-1)^{k} \quad \text { with } \alpha_{-2}=\frac{2}{D^{\prime \prime}(1)}=\frac{2}{\sigma^{2}} .
$$

Now removing the singularity at $z=1$, it turns out that

$$
f(z)=h(z)-\frac{\alpha_{-2}}{(1-z)^{2}}-\frac{\alpha_{-1}}{1-z}
$$

is analytic in $W=\{z:|z|<R\}$, therefore $f(z)=\sum_{k \geqslant 0} c_{k} z^{k}$ for $|z|<R$, since $R>1$, Cauchy's inequality yields $\left|c_{k}\right| \leqslant M(r) / r^{k}$ for any $r$ with $0<r<R$ and $M(r)=\sup _{|z|=r}|f(z)|$. Therefore the $c_{k}$ are geometrically decreasing.

Hence

$$
\sum_{k \geqslant 1} B_{k} z^{k}=\frac{\alpha_{-2}}{(1-z)^{2}}+\frac{\alpha_{-1}}{1-z}+\sum_{k \geqslant 0} c_{k} z^{k} \text { for }|z|<1
$$

expanding $\frac{\alpha_{-2}}{(1-z)^{2}}$ and $\frac{\alpha_{-1}}{1-z}$ in Taylor's series in the neighbourhood of 0 yields

$$
\sum_{n \geqslant 1} B_{n} z^{n}=\alpha_{-2} \sum_{n \geqslant 0}(n+1) z^{n}+\alpha_{-1} \sum_{n \geqslant 0} z^{n}+\sum_{n \geqslant 0} c_{n} z^{n}
$$

and at last we arrive at

$$
\begin{aligned}
& B_{0}=\alpha_{-1}+\alpha_{-2}+c_{0}=0, \\
& B_{k}=\alpha_{-1}+(k+1) \alpha_{-2}+c_{k} \quad \text { for } k \geqslant 1 .
\end{aligned}
$$

To carry out the following calculations, we recall some elementary relationships between the coefficients $a_{k}$ :

$$
\begin{aligned}
& -a_{-1}+\sum_{k \geqslant 1} \bar{a}_{k}=E(A)=0, \\
& \sum_{k \geqslant 1} k \bar{a}_{k}=\frac{1}{2} \sigma^{2} .
\end{aligned}
$$

Inserting (2.6) into (2.4) leads to

$$
\begin{aligned}
\frac{\pi_{N}(n)}{\pi_{N}(0)}= & +\alpha_{-1}\left(-a_{-1}+\sum_{j=1}^{n} \bar{a}_{j}\right)+\alpha_{-2}\left[(n+2) \sum_{k=1}^{n} \bar{a}_{k}-(n+1) a_{-1}-\sum_{k=1}^{n} k \bar{a}_{k}\right] \\
& +\sum_{j=1}^{n} c_{j} \bar{a}_{n-j+1}-c_{n} a_{-1} .
\end{aligned}
$$

Taking (2.7) and (2.8) into account, it turns out that

$$
\frac{\pi_{N}(n)}{\pi_{N}(0)}=1+\alpha_{-2}\left(a_{-1}-\frac{1}{2} \sigma^{2}\right)-\alpha_{-1} \sum_{j>n} \bar{a}_{j}+\alpha_{-2} \sum_{k>n}(k-n-2) \bar{a}_{k}+\sum_{j=1}^{n} c_{j} \bar{a}_{n-j+1}-c_{n} a_{-1} .
$$


Since $\alpha_{-2}=2 / \sigma^{2}$, then $1+\alpha_{-2}\left(a_{-1}-\frac{1}{2} \sigma^{2}\right)=2 a_{-1} / \sigma^{2}=k_{0}$ and finally we can claim

\section{Lemma 2.}

$$
\pi_{N}(n)=\left(k_{0}+u_{n}+v_{n}+w_{n}\right) \pi_{N}(0),
$$

where

$$
\begin{aligned}
& u_{n}=-\alpha_{-1} \sum_{j>n} \bar{a}_{j}, \\
& v_{n}=\alpha_{-2} \sum_{k>n}(k-n-2) \bar{a}_{k}, \\
& w_{n}=\sum_{j=1}^{n} c_{j} \bar{a}_{n-j+1}-c_{n} a_{-1} .
\end{aligned}
$$

Lemma 3. The three series $\sum_{n \geqslant 1}\left|u_{n}\right|, \sum_{n \geqslant 1}\left|v_{n}\right|, \sum_{n \geqslant 1}\left|w_{n}\right|$ are convergent.

Proof. It has been shown above that $D(z)$ and $f(z)$ are analytic in the disk $\{z:|z|<R\}$ for $R>1$. Taking $r$ with $1<r<R$, Cauchy's inequality shows that there exist two constants $M_{1}, M_{2}$ such that for any $k:\left|a_{k}\right| \leqslant M_{1} q^{k}$, $\left|c_{k}\right| \leqslant M_{2} q^{k}$ with $q=r^{-1}$.

In view of (2.10)-(2.12), it is seen that

$$
\begin{aligned}
& \left|u_{n}\right| \leqslant\left|\alpha_{-1}\right| \sum_{j>n} \sum_{k \geqslant j}\left|a_{k}\right| \leqslant M_{1}\left|\alpha_{-1}\right|(1-q)^{-1} q^{n+1}, \\
& \left|v_{n}\right| \leqslant\left|\alpha_{-2}\right| \sum_{k>n} k \bar{a}_{k} \leqslant M_{1}(1-q)^{-1}\left|\alpha_{-2}\right| \sum_{k>n} k q^{k} \leqslant M_{1}(1-q)^{-2}\left|\alpha_{-2}\right| q^{n}\left(n+1+q(1-q)^{-1}\right), \\
& \left|w_{n}\right| \leqslant M_{2} a_{-1} q^{n}+n M_{1} M_{2}(1-q)^{-1} q^{n+1} .
\end{aligned}
$$

Since $q=r^{-1}<1$, the proof is achieved.

As a consequence of (2.9), $\pi_{N}$ can be expressed as

$$
\pi_{N}(n)=\left(k_{0}+z_{n}\right)\left[1+N k_{0}+\sum_{1}^{N} z_{j}\right]^{-1}, \quad \text { with } z_{n}=u_{n}+v_{n}+w_{n} \text { and } \sum_{n \geqslant 1}\left|z_{n}\right|<+\infty .
$$

\subsection{Proof of Property 1}

\subsubsection{Proof of (i)}

Letting $s_{N}=\sum_{1}^{N} z_{n}, \sigma_{N}=\sum_{1}^{N}\left|z_{n}\right|$, formula (2.13) shows that

$$
\begin{aligned}
& \left|\pi_{N}(0)-\frac{1}{N+1}\right|=\left|\frac{1}{1+N k_{0}+s_{N}}-\frac{1}{N+1}\right|=\frac{\left|N\left(k_{0}-1\right)+s_{N}\right|}{(N+1)\left(1+N k_{0}+s_{N}\right)}, \\
& \left|\pi_{N}(n)-\frac{1}{N+1}\right|=\left|\frac{k_{0}+z_{n}}{1+N k_{0}+s_{N}}-\frac{1}{N+1}\right|=\frac{\left|k_{0}-1+(N+1) z_{n}-s_{N}\right|}{(N+1)\left(1+N k_{0}+s_{N}\right)} \quad \text { for } 1 \leqslant n \leqslant N .
\end{aligned}
$$

Therefore

$$
\Delta_{N}=\sum_{0}^{N}\left|\pi_{N}(n)-\frac{1}{N+1}\right| \leqslant \frac{2 N\left|k_{0}-1\right|+(2 N+1) \sigma_{N}}{(N+1)\left(1+N k_{0}+s_{N}\right)}
$$

and from $\sum_{n \geqslant 1}\left|z_{n}\right|<+\infty$, it turns out that $\varlimsup \Delta_{N}<+\infty$. 
2.2.2. Proof of (ii)

From (2.13),

$$
\Delta_{N}=\sum_{0}^{N}\left|\pi_{N}(n)-\frac{1}{N+1}\right| \geqslant\left|\pi_{N}(0)-\frac{1}{N+1}\right|=\frac{\left|N\left(k_{0}-1\right)+s_{N}\right|}{(N+1)\left(1+N k_{0}+s_{N}\right)},
$$

therefore

$$
N \Delta_{N} \geqslant \frac{N^{2}}{(N+1)\left(1+N k_{0}+s_{N}\right)}\left|k_{0}-1+\frac{s_{N}}{N}\right|,
$$

since $\sum_{n \geqslant 1}\left|z_{n}\right|<+\infty$, we get $\underline{\lim } N \Delta_{N} \geqslant\left|k_{0}-1\right| / k_{0}$, hence $k_{0}=1$. From $k_{0}=2 a_{-1} / \sigma^{2}=1$ and $E\{A\}=0$, (2.7) and (2.8) entail $a_{-1}=\sum_{k \geqslant 1} k^{2} a_{k}$ and $a_{-1}=\sum_{k \geqslant 1} k a_{k}$ leading to $a_{k}=0$ for $k \geqslant 2$. Therefore $a_{-1}=a_{1}$ and $a_{0}+a_{-1}+a_{1}=1$ but in this case it is readily checked that the stationary distribution is uniform for any $N: \pi_{N}(n)=\frac{1}{N+1}, 0 \leqslant n \leqslant N$, the proof is achieved.

Remark 1. These proofs entail that for any $p \geqslant 1$ the following properties hold true:

(i) $\varlimsup_{\lim } N^{p} \sum_{0}^{N}\left|\pi_{N}(n)-\frac{1}{N+1}\right|^{p}<+\infty$.

(ii) If $\underline{\lim } N^{p} \sum_{0}^{N}\left|\pi_{N}(n)-\frac{1}{N+1}\right|^{p}=0$, then for any $N, \pi_{N}$ is uniform on $\{0,1,2, \ldots, N\}$.

Remark 2. This result remains true if (AS1), (AS3) are changed into

$\left(\mathrm{AS}^{\prime}\right) P(A>1)=0, a_{1}>0, a_{0}+a_{1}<1$.

$\left(\mathrm{AS}^{\prime}\right) \exists r>1, E\left(r^{-A}\right)<+\infty$.

In fact, assuming that $\left(\mathrm{AS}^{\prime}\right),\left(\mathrm{AS}^{\prime}\right)$ are true, it can be seen from $(1.1)$ that $\left(Y_{N}(t) ; t \geqslant 0\right)$, with $Y_{N}(t)=$ $N-X_{N}(t)$ obeys the relation:

$$
Y_{N}(t+1)=\min \left(N, \max \left(0, Y_{N}(t)-A(t+1)\right)\right)
$$

therefore Property 1 applies with the limiting distribution $\mu_{N}$ of $\left(Y_{N}(t) ; t \geqslant 0\right)$, that is

$$
\varlimsup N \sum_{0}^{N}\left|\mu_{N}(n)-\frac{1}{N+1}\right|<+\infty .
$$

If

$$
\underline{\lim } N \sum_{0}^{N}\left|\mu_{N}(n)-\frac{1}{N+1}\right|=0
$$

then $\mu_{N}$ is uniform on $\{0,1,2, \ldots, N\}$.

Since $\mu_{N}(n)=\pi_{N}(N-n)$ the conclusions of Property 1 remain valid for $\pi_{N}$.

\subsection{Proof of Theorem 2}

Let $F_{N}(x)=\pi_{N}([0, N x])$ and as usual $\|f\|_{\infty}=\sup _{x}|f(x)|$. First, we observe that

$$
N\left\|F_{N}-G\right\|_{\infty} \geqslant N F_{N}(0)=N \pi_{N}(0)=\frac{N}{1+N k_{0}+s_{N}}
$$


in view of formula (2.13), therefore

$$
\begin{aligned}
& \underline{\lim } N\left\|F_{N}-G\right\|_{\infty} \geqslant k_{0}^{-1}=\frac{\sigma^{2}}{2 a_{-1}} . \\
& \left|F_{N}(x)-x\right|=\left|\sum_{k=0}^{[N x]} \pi_{N}(k)-x\right| \leqslant \sum_{k=0}^{k=[N x]}\left|\pi_{N}(k)-\frac{1}{1+N}\right|+\left|\frac{[N x+1]}{N+1}-x\right|,
\end{aligned}
$$

now

$$
\left|\frac{[N x+1]}{N+1}-x\right| \leqslant \frac{2}{N+1}
$$

for $0 \leqslant x \leqslant 1$.

Property 1 entails that $\overline{\lim } N\left\|F_{N}-G\right\|_{\infty}<+\infty$, which completes the proof.

\subsection{Proof of Theorem 3}

For $p \geqslant 1$, let's consider

$$
\left\|F_{N}-G\right\|_{p}^{p}=\int_{-\infty}^{+\infty}\left|F_{N}(x)-G(x)\right|^{p} \mathrm{~d} x=\int_{0}^{1}\left|F_{N}(x)-x\right|^{p} \mathrm{~d} x,
$$

obviously, Theorem 2 gives $\overline{\lim } N\left\|F_{N}-G\right\|_{p}<+\infty$. Noticing that

$$
\int_{k / N}^{k+1 / N}\left|F_{N}(x)-x\right|^{p} \mathrm{~d} x \geqslant \frac{1}{(p+1) 2^{p} N^{p+1}},
$$

it turns out that

$$
\underline{\lim } N\left\|F_{N}-G\right\|_{p} \geqslant \frac{1}{2}(p+1)^{-\frac{1}{p}} .
$$

\subsection{Examples}

Example 1. Let's consider the case when the d.f of the r.v $A$ is geometric with parameter $\frac{1}{2}$, i.e. $a_{k}=2^{-(k+2)}$ for $k \geqslant-1$. Then

$$
E\{A\}=0, \quad E\left\{A^{2}\right\}=2, \quad k_{0}=\frac{2 a_{-1}}{E\left\{A^{2}\right\}}=\frac{1}{2}, \quad h(z)=\frac{z}{D(z)}=-1+\frac{1}{(z-1)^{2}},
$$

therefore $\alpha_{-1}=0$ and $c_{n}=0$ for $n \geqslant 1,(2.10)$ and (2.12) show that $u_{n}=w_{n}=0$. As for $v_{n}=\alpha_{-2} \sum_{k>n}(k-$ $n-2) \bar{a}_{k}$, it can be checked that $v_{n}=0$, hence $z_{n}=0,(2.13)$ gives $\pi_{N}(n)=\frac{1}{2} \pi_{N}(0)$. Therefore

$$
\Delta_{N}=\sum_{0}^{N}\left|\pi_{N}(n)-\frac{1}{N+1}\right|=\frac{2 N}{(N+1)(N+2)}
$$

and $\lim _{N} N \Delta_{N}=2$, which totally agrees with the result of Property 1 .

In fact, a glance at $P_{N}$ shows that $(2,1,1, \ldots, 1)$ is a left eigenvector, consequently we have $\pi_{N}(0)=\frac{2}{N+2}$ and $\pi_{N}(n)=\frac{1}{N+2}$ for $1 \leqslant n \leqslant N$, leading to $\lim _{N} N \Delta_{N}=2$. 
Example 2 (The $M / G I / 1 / N$ queueing system). We consider a $M / G I / 1 / N$ queueing system, $(N-1$ waiting spaces). Arrival occur according to a Poisson process with rate $\lambda$, the service times $S_{1}, S_{2}, \ldots$ are i.i.d r.v's having a c.d.f $F$ with mean $\mu^{-1}$ and standard deviation $\sigma$. It is assumed that $\lambda=\mu$ and that $E\left\{\mathrm{e}^{\theta S}\right\}<+\infty$ for a $\theta>0$.

As usual, let $Y_{N}(m)$ the number of customers left in the system just after the departure of the mth customer. It is known that the embedded process $\left(Y_{N}(m)\right)_{m \geqslant 1}$ is a Markov chain obeying the recursive relation

$$
Y_{N}(m+1)=\min \left\{N-1, \max \left(0, Y_{N}(m)-1\right)+A(m+1)\right\} \quad \text { for any } m \geqslant 1,
$$

where $A(m)$ denotes the number of arriving customers while the $m$ th customer is being served, with:

$$
a_{k}=P(A(m)=k)=\int_{0}^{+\infty} \mathrm{e}^{-\lambda t} \frac{(\lambda t)^{k}}{k !} \mathrm{d} F(t) \quad \text { for } k \geqslant 0 .
$$

Due to the finite capacity, for $\rho=\lambda / \mu=1, \pi_{N}(j)=\lim _{m} P\left(Y_{N}(m)=j\right)$ exists.

Setting $Z_{N}(m)=\max \left\{0, Y_{N}(m)-1\right\}$ in $(2.15)$ we arrive at

$$
Z_{N}(m+1)=\min \left\{N-2, \max \left\{0, Z_{N}(m)+B(m+1)\right\}\right\} .
$$

Thus $\left(Z_{N}(m)\right)$ is a two-sided regulated random walk with a lower barrier at 0 and an upper barrier at level $N-2$, with increment $B(m)=A(m)-1$. In view of the assumptions on the service time stated above, it can be easily checked that the increment $B(m)$ fulfills (AS1), (AS2), (AS3). Therefore, Property 1 applies and the stationary distribution $\eta_{N}=\left(\eta_{N}(j)\right)_{0 \leqslant j \leqslant N-2}$ of $\left(Z_{N}(m)\right)$ obeys the relation

$$
\sum_{j=0}^{N-2}\left|\eta_{N}(j)-\frac{1}{N-1}\right|=\mathrm{O}\left(\frac{1}{N}\right)
$$

From $\pi_{N}(j)=\eta_{N}(j-1)$ for $2 \leqslant j \leqslant N-1$ and $\pi_{N}(0)+\pi_{N}(1)=\eta_{N}(0)$ it turns out that

$$
\sum_{n=0}^{N-1}\left|\pi_{N}(n)-\frac{1}{N}\right|=\mathrm{O}\left(\frac{1}{N}\right) \text {. }
$$

That is, for $N$ large the stationary distribution is "almost" uniform on $\{0,1,2, \ldots, N-1\}$.

Example 3 (The $G I / M / 1 / N$ queueing system). We consider a $G I / M / 1 / N$ queueing system $(N-1$ waiting spaces). Customers arrive at epochs $0<t_{1}<t_{2}<\cdots<t_{m}<\cdots$ where the inter-arrival times $T_{n}=t_{n}-t_{n-1}, n \geqslant 1$ $\left(t_{0}=0\right)$ are i.i.d r.v's having a nonlattice c.d.f $H$ with mean $\lambda^{-1}$. The service times $S_{1}, S_{2}, \ldots$ are i.i.d r.v's having a common exponential d.f with mean $\mu^{-1}$. It is assumed that $\lambda=\mu$ and that $E\left\{\mathrm{e}^{\theta T}\right\}<+\infty$ for a $\theta>0$. Let $Y_{N}(m)$ the number of customers in the system seen upon arrival by the $m$ th customer. Due to the finite capacity, $\pi_{N}(j)=\lim _{m} P\left(Y_{N}(m)=j\right)$ exists.

$\left(Y_{N}(m)\right)_{m \geqslant 1}$ has the same probability distribution as the Markov chain $\left(\tilde{Y}_{N}(m)\right)_{m \geqslant 1}$ defined by the recursive relation:

$$
\tilde{Y}_{N}(m+1)=\max \left\{0, \min \left(\tilde{Y}_{N}(m), N-1\right)+1-\tilde{A}(m+1)\right\} \quad \text { for any } m \geqslant 1,
$$

$\tilde{A}(m+1)$ being the number of Poisson events during the time interval $\left[t_{m}, t_{m+1}\right)$.

Setting $Z_{N}(m)=\max \left\{0, N-1-\tilde{Y}_{N}(m)\right\}$ in $(2.18)$ we arrive at

$$
Z_{N}(m+1)=\min \left\{N-1, \max \left\{0, Z_{N}(m)+\tilde{A}(m+1)-1\right\}\right\} .
$$

Thus $\left(Z_{N}(m)\right)$ is a two-sided regulated random walk with a lower barrier at 0 and an upper barrier at level $N-1$, with increment $B(m)=\tilde{A}(m)-1$. Again, the assumptions made on the inter-arrival times entail that 
the increment $B(m)$ fulfills the (AS1), (AS2), (AS3). Therefore, Property 1 applies and the stationary distribution $\eta_{N}=\left(\eta_{N}(j)\right)_{0 \leqslant j \leqslant N-1}$ of $\left(Z_{N}(m)\right)$ obeys the relation

$$
\sum_{j=0}^{N-1}\left|\eta_{N}(j)-\frac{1}{N}\right|=\mathrm{O}\left(\frac{1}{N}\right) .
$$

From $\pi_{N}(j)=\eta_{N}(N-j-1)$ for $0 \leqslant j \leqslant N-2$ and $\pi_{N}(N)+\pi_{N}(N-1)=\eta_{N}(0)$ again, it turns out that

$$
\sum_{n=0}^{N}\left|\pi_{N}(n)-\frac{1}{N+1}\right|=\mathrm{O}\left(\frac{1}{N}\right) .
$$

\section{References}

[1] S. Asmussen, Applied Probability and Queues, Wiley, 1987.

[2] A.A. Borovkov, Asymptotic Methods in Queuing Theory, Wiley, 1984.

[3] D. Gibson, E. Seneta, Monotone infinite stochastic matrices and their augmented truncations, Stochastic Process. Appl. 24 (1987) $287-292$.

[4] J.M. Harrison, Brownian Motion and Stochastic Flow Systems, Krieger Publishing Company, Malabar, FL, 1990.

[5] J.F. Hayes, Modeling and Analysis of Computer Communications Networks, Plenum Press, New York, 1984.

[6] D.P. Heyman, W. Whitt, Limits of queues as the waiting room grows, QUESTA 5 (1989) 381-392.

[7] D.P. Heyman, Approximating the stationary distribution of an infinite stochastic matrix, J. Appl. Probab. 28 (1991) 96-103.

[8] V.V. Kalashnikov, Mathematical Methods in Queuing Theory, Kluwer Academic, 1994.

[9] V.S. Korolyuk, Boundary Problems for Compound Poisson Process, Naukova Dumka, Kiev, 1975.

[10] J. Mehdi, Stochastic Models in Queueing Theory, Academic Press, 1991.

[11] N.U. Prabhu, Stochastic Storage Processes, Springer-Verlag, New York, 1980.

[12] F. Simonot, Sur l'approximation de la distribution stationnaire d'une chaîne de Markov stochastiquement monotone, Stochastic Process. Appl. 56 (1995) 133-149.

[13] F. Simonot, Y.Q. Song, Characterization of convergence rates for the approximation of the stationary distribution of infinite monotone stochastic matrices, J. Appl. Probab. 33 (1996) 974-985.

[14] F. Simonot, Moment based approximation for the stationary distribution of a random walk in $Z_{+}$with an application to the $M / G / 1 / n$ queueing system, J. Appl. Probab. 37 (2000) 290-299.

[15] F. Simonot, A.D. Manita, Convergence time to equilibrium for nonhomogeneous stochastically monotone Markov chains, Preprint 9 , Lyapounov French-Russian Institute, Moscow, 2001.

[16] D. Stoyan, Comparison Methods for Queues and Other Stochastic Models, Wiley, 1983.

[17] H.C. Tijms, Stochastic Modelling and Analysis, Wiley, 1986.

[18] R.L. Tweedie, Truncation Approximations of Invariant Measures for Markov Chains, J. Appl. Probab. 35 (1998) 517-536.

[19] D. Wolf, Approximation of the invariant probability distribution of an infinite stochastic matrix, Adv. Appl. Probab. 12 (1980) $710-726$. 